\title{
An Innovative Design of Automatic Feeding System for Fuel Injection Pump Plunger
}

\author{
Liang Hana, Xiang Lib, Kaige Yanc \\ School of Mechanical Engineering, Southeast University, Jiangning \\ District, Nanjing 211189, China \\ amelhan@seu.edu.cn,b1028708610@qq.com,ckaige_yan@163.com
}

\begin{abstract}
Fuel injection pump plunger is one of the most important parts of diesel engine, and its processing quality and productivity reflect a country's industrialization and automation level. An automatic feeding system for fuel injection pump plunger grinding process is proposed and designed, which mainly includes a vibratory bowl feeder, a linear vibratory feeder, a pneumatic manipulator and a fiber optic sensor. Through the system, the plungers can realize arrangement, direction judgment, direction adjustment and linear transmission. This paper focuses on vibratory transmission principle, pneumatic system and structure of the manipulator and direction judgment with fiber optic sensor. This automatic feeding system overcomes some of disadvantages of the traditional devices and increases the automation level of plunger production.
\end{abstract}

Keywords: Automatic Feeding; Fuel Injection Pump Plunger; Pneumatic Manipulator

\section{Introduction}

The diesel engine is a device which turns chemical energy into mechanical energy [1]. Compared with the gasoline, diesel fuel has better power performance; but it must be atomized and mixed to burn effectively because of its high spontaneous combustion temperature and low volatilization. When the atomization and mixture are uncontrolled, it is easy to generate non-homogeneous combustion, which may result in violent and unstable combustion. Because of these characteristics, the diesel engine injection system has become the key technology to improve fuel efficiency and reduce pollution. Fuel injection pump is the core component of diesel engine and the plunger matching parts are the most precise parts of injection pump, which is composed of a plunger and a sleeve. The Plunger moves in the sleeve completing the process of fuel absorbing and injecting. So the structure precision and quality are very significant to injection pressure and fuel uniformity. Also, the quality of plunger has a great influence on reliability and performance of diesel engine[3-4]. 
The manufacturing quality of plunger directly affects the performance of fuel injection pump. And its production also reflects the industrial automation level of a country. In the processing, plungers need to be transmitted to grinder where two important surfaces of plunger are grinded. The traditional equipment not only is expensive but also has short service life and high error ratio, so it is necessary to develop new automation equipment, which can transmit the plungers into the grinder quickly and stably. This paper proposes a novel design of the automatic feeding device for fuel injection pump plungers.

\section{Overall Design of Plunger Automatic Feeding System}

Automatic loading and unloading systems are indispensable in automatic machines, which mainly include arrangement and orientation devices, transmitting devices, clamping and unloading mechanisms [5]. The most important parts are automatic arrangement and orientation mechanisms. Automatic feeding process of plunger grinding comprises storage, arrangement, orientation, linear transmission and clamping, etc. As shown in Fig.1, the automatic feeding device includes vibratory bowl feeder, pneumatic manipulator, linear vibratory feeder, sensors and the base.

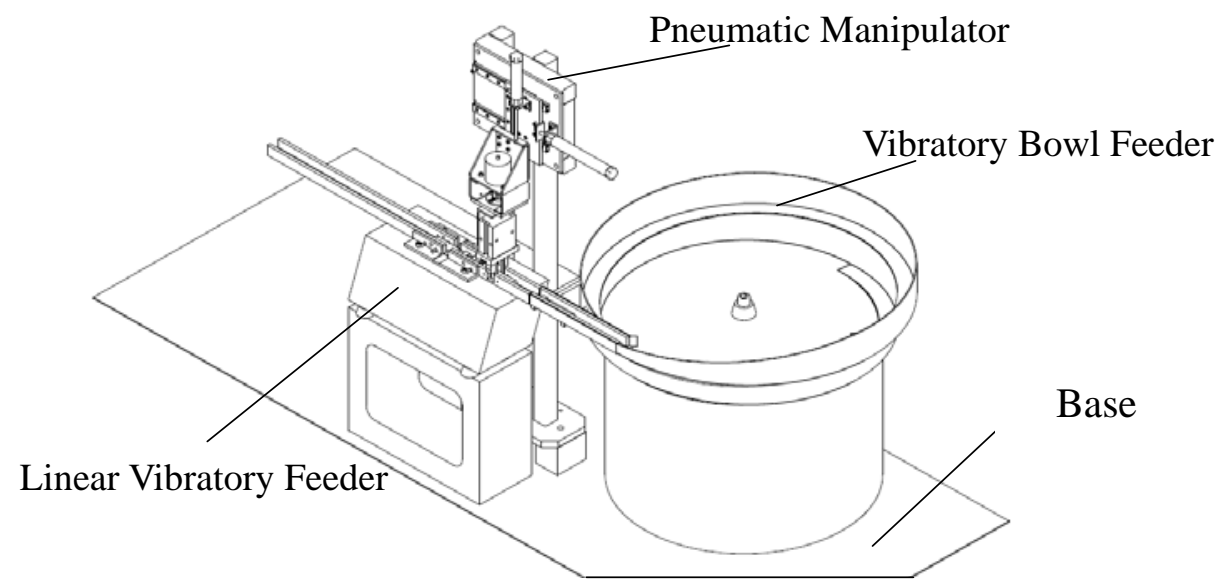

Fig.1 Structure of Plunger Automatic Feeding System

First of all, plungers are stored, oriented and transmitted in the vibratory bowl feeder, and then the plungers are transmitted to front part of linear feeder. Because of the requirement of grinder, plunger must be transmitted in the linear feeder with big end forward. Secondly, block and the optical fiber sensor are fixed in the linear vibration feeder. When the plunger is stopped by the block and detected by a position sensor, the optical fiber sensor will judge the direction of plunger. Pneumatic manipulator can grasp plunger and take it to move in vertical motion, horizontal motion and $180^{\circ}$ rotation. When the fiber sensor detects that the direction of a plunger is correct, pneumatic manipulator will grasp and take it to the next station of linear feeder. When the direction is wrong, the plunger will be rotated $180^{\circ}$ before being moved to the next station. Thirdly, the linear 
vibratory feeder transmits plungers to the next station, waiting for the operation of clamping manipulator.

\section{Principle of Electromagnetic Vibratory Feeder}

Compared with other feeding systems, vibratory feeder has advantages of high feeding efficiency, good stability and reliability, simple structure and good compatibility. In the transmission, parts can be orientated easily by orientation mechanisms. Hence, vibratory feeders are widely used in automatic manufacturing and assembly [6].

Structure of electromagnetic vibratory bowl feeder is shown in Fig.2. When the electromagnet is powered by alternating current, the electromagnetic force vibrates the bowl up and down with high-frequency and micro-amplitude, which makes the bowl vibrate torsionally by the inclined leaf springs. Then work-pieces can move upward along the spiral groove. And in the transmission, work-pieces can realize the desired state through orientation mechanisms.

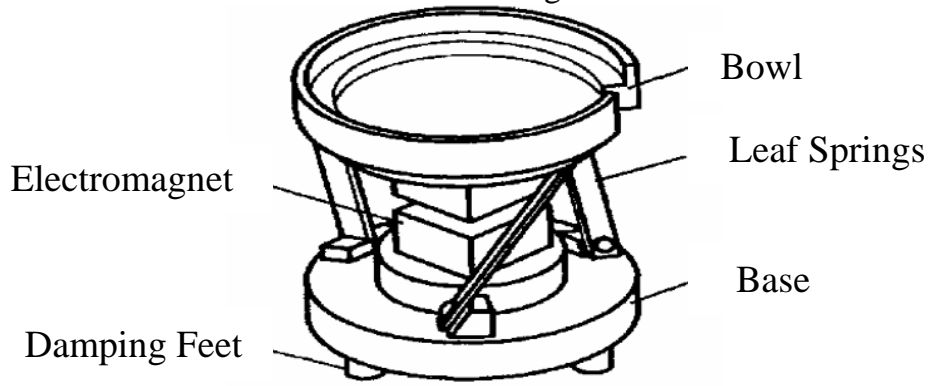

Fig.2 Structure of Electromagnetic Vibratory Bowl Feeder

\section{Design of Pneumatic Manipulator}

With the development of electronic technology and wide application of computers, the research of robots has become a novel technology and developed rapidly, which also promotes the development of manipulator combining of mechanization and automation [7-9].

Compared with other manipulators, pneumatic manipulator has advantages of low price, simple structure, high ratio of power and volume, no pollution and strong anti-interference ability, etc. [10] Pneumatic manipulator in the plunger automatic feeding system mainly includes the horizontal cylinder, vertical cylinder, rotary cylinder, the pneumatic gripper, linear guide rail, magnetic base and support bracket, which can realize grasp and moving in vertical motion, horizontal motion and $180^{\circ}$ rotation. Magnetic bases ensure pneumatic manipulator being fixed and its position is convenient to adjust and move. The structure and pneumatic system is shown in Fig.3. 


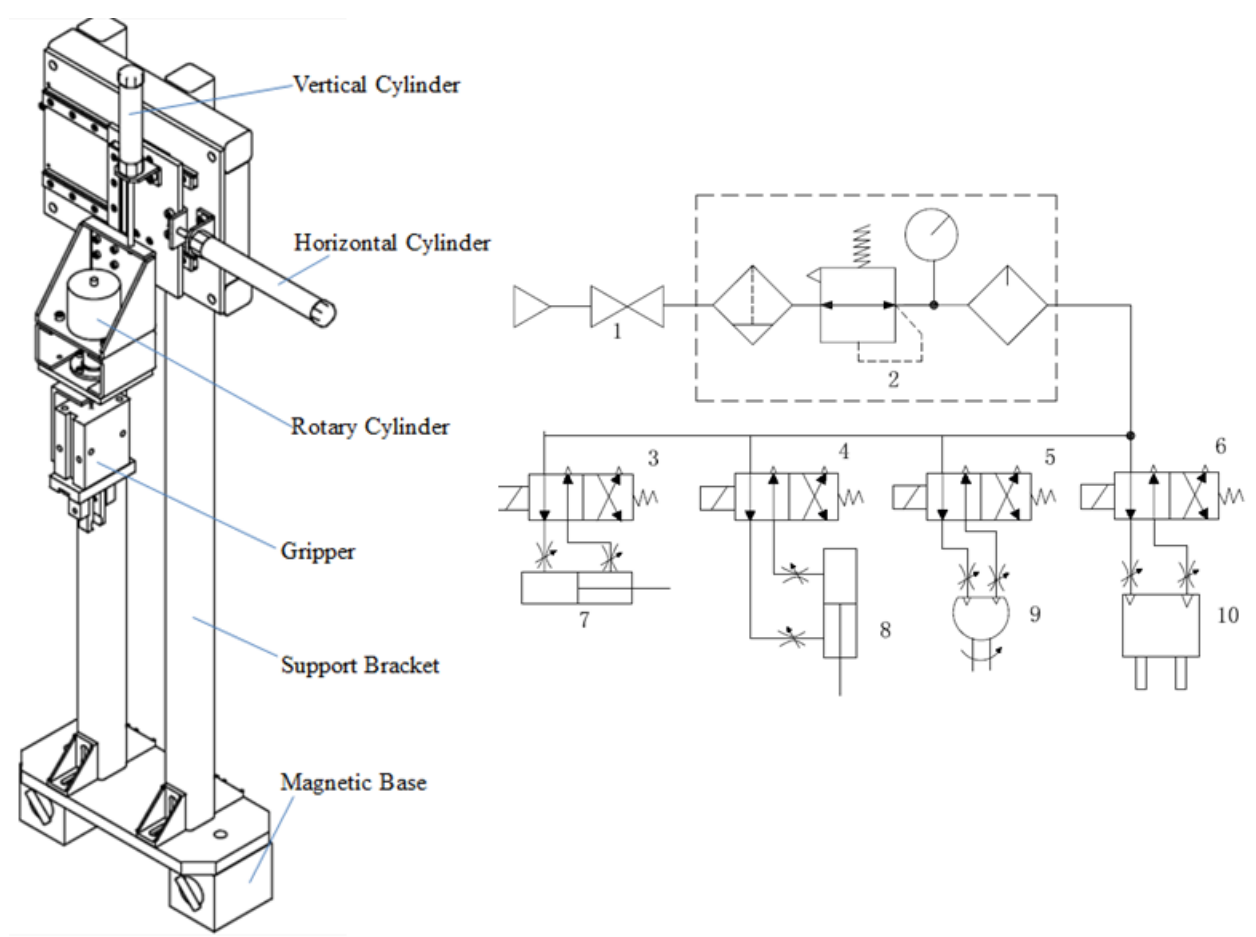

Fig.3 Structure and Pneumatic System of the Manipulator

\section{Plunger Direction Judgment with Fiber Optic Sensor}

Fiber optic sensing technology is an emerging technology beginning in 1970s. Fiber optic sensors are widely used because of their superior performances, such as small size, corrosion preventing, high sensitivity and wide measurement range [11-12]. 


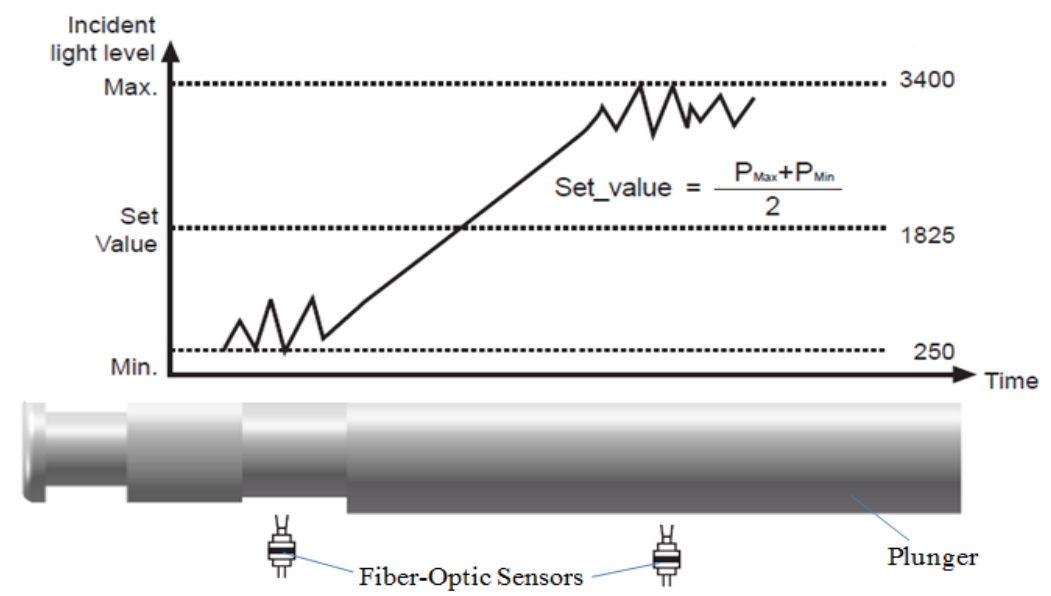

Fig.4 Principle of Direction Judgment with Fiber Optic Sensor

Autonics-BF5 fiber optic sensor is used to judge the directions of the plungers in this automatic feeding system. The principle is shown in Fig.6. When the light emitted from the optical fiber sensor exposes to the small diameter, the incident light level is relatively lower than the set value because the distance between fiber optic sensor head and the reflecting surface is long. When light exposes to the large diameter, distance between fiber optic sensor head and the reflector is short, so the incident light level is relatively higher than the set value. In conclusion, the sensor has a different output to distinguish the directions of the plunger.

\section{Conclusion}

The quality of plunger affects the performance of fuel injection pump directly, and it is also the key technology to improve fuel efficiency and reduce pollution of diesel engine. Research on automatic feeding system of plunger, not only improves the uniformity of manufacturing and production efficiency, but also avoids the errors caused by operators and reduces the labor intensity. The automatic feeding device designed in this paper combines the vibratory transmission, pneumatic manipulator and fiber optic sensing technology. It has advantages of flexible structure, convenient processing and adjustment, high efficiency and accuracy, and it overcomes some disadvantages of traditional plunger feeding device, so it has good research value and application prospect.

\section{Acknowledgement}

This work is supported by NSFC, project No.: 51275087.

\section{Reference}


[1] Jiang Ying-shuo, Wang Dong-hua. Functional reliability comparison evaluation for fuel-ejecting pumps of diesel engines [J]. Journal of Shenyang Jianzhu University, Vol.22, No.4, 2006

[2] Jiang Yuehua. Research of heat treatment technics of the injection pump camshaft [D]. Shanghai Jiao Tong University, Shanghai, 2007

[3] Wang Jianguo. New grinding technology for spiral fuel supply edge of fuel injection pump's plunger [J]. Diesel Engine, Vol.32, No.3, 2010

[4] Jiang Genghong. On the mechanism and Precaution of fuel pump plunger cavitation [J]. Ship and Ocean Engineering, Vol.40, No.3, 2011

[5] Liu Chunlin. Design and research of machine automatic feeding mechanism [J]. Machinery, Vol.51, No.585, 2013

[6] Ding Xiaodong. Structural design of vibration hopper [J]. Electro-Mechanical Engineering, Vol.23, No.6, 2007

[7] Zhang Jinping. The hydraulic drive system based on automatic feeding manipulator design [J].Manufacturing Automation, Vol.35, No.11, 2013

[8] Yang Zhenqiu. The research and application of a simplified accurate positioning pneumatic manipulator [J]. HuaZhong University of Science and Technology, Wuhan, 2006

[9] Song Zhifeng. Control system design of pneumatic manipulator [J]. Mechanical and Electrical Technology, 2011

[10] Wang Denggui. The Control system for pneumatic manipulator [J]. Techniques of Automation and Applications, Vol.28, No.8, 2009

[11] Gu Bobo. Some optical sensors based on novel structured fibers and their application [D]. ZheJiang University, 2012

[12] Zhang Sen, Liu Menghua. Development and applications of fiber-optic sensor [J]. Study Optical Communication, No.141, 2007 